Suprihati and Yunus, Afr., J. Infect. Dis. (2018) 12(S): 104-110

https://doi.org/10.2101/Ajid.v12i1S.15

\title{
EVALUATION OF THE ANTIGENICITY AND IMMUNOGENICITY OF Eimeria tenella BY REPRODUCTIVE INDEX AND HISTOPATHOLOGICAL CHANGES OF CECAL COCCIDIOSIS VIRULENT LIVE VACCINE IN BROILER CHICKENS
}

\author{
Endang Suprihati ${ }^{1 *}$, Muchammad Yunus ${ }^{2}$ \\ ${ }^{1,2}$ Department of Veterinary Parasitology, Faculty of Veterinary Medicine, Airlangga University, Surabaya, \\ Indonesia
}

*Corresponding Author Email: esuprihati@yahoo.co.id

\begin{abstract}
$\underline{\text { Article History }}$
Received: March. 15, 2017

Revised Received: Oct. 18, 2017

Accepted: Oct. 19, 2017

Published Online: March. 07, 2018
\end{abstract}

\begin{abstract}
Background: The development of vaccine to control coccidiosis caused by Eimeria tenella (E. tenella) in chickens is intensifying because of the increasing threat of drug resistance to anticoccidial agents. It is important, therefore, to develop a reliable standard method for the assessment of vaccine afficacy particularly antigenicity and immunogenicity become crucial. Evaluation of E. tenella antigenicity and immunogenicity to some low doses can be reflected by reproductive index and histopathological changes.

Materials and Methods: The complete random design of research was used in this study. Sixty of two weeks old broilers were divided into four groups and each group composed 15 replications. The group 1 was chicken group without virulent $E$. tenella oocyst inoculation. The group 2, 3 and group 4 were chicken group inoculated with virulent E. tenella oocyst at doses of $1.0 \times 10^{2}, 2.0 \times 10^{2}, 3.0 \times 10^{2}$, respectively. Then all chicken groups were challenged with E. tenella oocyst at doses of $1.0 \times 10^{3}$. Observation of research that represented antigenicity and immunogenicity was clinical sign, reproductive index, histopathological changes.

Results: On virulent E. tenella inoculation step, some clinical signs such as appetite, weakness, and diarrhea were very slight on all chicken groups. While on challenge test step, there were no clinical signs of all chicken groups except the group 1. For the reproductive index of virulent E. tenella inoculation step, there were no significantly differences in all chicken groups except the group 1. As reproductive index, the same result pattern was seen for histopathological changes.

Conclusion: The low number virulent $E$. tenella had low reproductive index and few histopathological changes effect that represents a promising strategy to prevent cecal coccidiosis in chickens.
\end{abstract}

Key words: antigenicity, E.tenella, histopthological changes, immunogenicity, reproductive index.

\section{Introduction}

Chicken coccidiosis is one of the most economically devastating diseases of poultry industry. Worldwide, the loss due to chicken coccidiosis cover low productivity, mortality, prophylaxis and treatment estimated more than 3 billion US dollars, annually (Dalloul, Lillehoj, 2006). One of pathogenic Eimeria species is E. tenella that cause cecal coccidiosis. On the other hand, the fact that on the poultry industry in the control of coccidiosis is mostly associated with the use of routinely anti-coccidiosis (coccidiostat). To overcome these conditions the option to conduct immunization approach against chicken in controlling the disease more intensive and planned indispensable. Immunization against chickens to control coccidiosis is more promising in avoiding or decreasing the reliance on the use of chemicals and other coccidiostat.

In the beginning, the immunization approach to cecal coccidiosis can be used by assessment of host response on antigen exposure. On the other hand evaluation of antigenicity of antigen of cecal coccidiosis can be done by assessment of reproductive index and/or multiplication index of antigen proliferation in site infection. Whereas histomorphology of cecum as site of endogenous development of parasite and the damage caused in initial infection is one of assessment to antigenicity. Furthermore, evaluation of immunogenicity of antigen of cecal coccidiosis can be done to assess that antigen in inducing 104 
protective immunity particularly when host is challenged. Whereas level of protective immunity can be expressed by reproductive index and/or multiplication index of proliferation and damage caused of parasite administered in challenge test.

Histomorphology of the cecum also reliably assesses the tissue architecture and/or featuring alteration integrity of cecum significantly supporting clinical diagnosis. Numerous reports demonstrate that sequential exposure of the host to Eimeria sp. stimulates a immune response that is capable of protecting hosts from a challenge inoculation. Acknowledging the compartmentalisation of immune responses underlines the importance of local events in the context of response on parasite exposure.

Even though a consent about major aspects to be evaluated that derive from experiences in pathology, diagnosis of coccidiosis is based on comparison of clinical features, gut pathology in the host, and parasite properties such as morphology of different parasite stages in fecal material or intestine and pre-patent period (Haug et al, 2007). Development of intracellular parasite such as genus Eimeria has a complex lifecycle, where it passes through asexual and sexual stages, so that observation of intracellular development this parasite is needed to study some potential development stages for biological control of coccidiosis. The present study was designed to evaluate antigenicity and immunogenicity by reproductive index and histomorphologically intracellular endogenous development of E. tenella in chicks by repeated infection in order to know the presence of development of protective immunity.

\section{Materials and Methods}

Cecal coccidiosis suspected to be naturally infected were collected from non commercial different poultry farms in Surabaya district. They were opened by giving longitudinal incision and the collected contents were examined by direct microscopy (Soulsby, 1986). The contents from positive samples were placed in $2.5 \%$ potassium dichromate $\left(\mathrm{K}_{2} \mathrm{Cr}_{2} \mathrm{O}_{7}\right)$ solution to induce sporulation at $28^{\circ} \mathrm{C}$ for 4-7 days. This strain was propagated in chicks to meet isolate needs of this study. This isolate had been stored in $2.5 \%$ potassium dichromate at $4^{\circ} \mathrm{C}$ until used.

A total of 60 pathogen-free male broiler chicks (CP 707) were purchased from poultry supplier Co., Surabaya. At 14 days old housed in clean metal cages and fed with a standard diet without coccidiostat and tap water ad libitum in an airconditioned room $\left(23 \pm 1^{\circ} \mathrm{C}\right)$, under conventional conditions with a 12:12 hr, light: dark cycle. They were kept as outlined in the "Guide for the Care and Use of Laboratory Animals" by The Faculty of Veterinary Medicine, Airlangga University.

All sixty pathogen-free male broiler chicks at two weeks old were divided into four groups and each group composed 15 replications. The group 1 was chicken group without infected orally virulent E. tenella oocyst. The group 2, 3 and group 4 were chicken group administered orally with virulent E. tenella oocyst at low doses of $1.0 \times 10^{2}, 2.0 \times 10^{2}, 3.0 \times 10^{2}$, respectively. At 5 days post infection (pi), five chickens of each group were sacrificed to observe morphological endogenous development of parasites by histopathological changes examination in primary infection, while the pattern of daily and total oocyst production calculation for expressing reproductive index was done at 6 to 12 days post infection on other infected chickens. Two weeks post infection of primary infection, all remaining chickens of each groups challenged with E. tenella oocyst with doses of $1.0 \times 10^{3}$. Then 5 days post challenge, five chickens of each groups were sacrificed to observe morphological endogenous development of parasites by histopathological changes examination in challenge infection, whereas the pattern of daily and total oocyst production counted at 6 to 12 days post challenge infection on five other infected chickens. Fecal materials were collected from 6 to 12 days post-infection of primary as well as challenge infection, and the number of oocysts per day was assessed using a MacMaster method counting chamber. Total oocyst numbers were calculated and histopathological examinations were evaluated as describe by Yunus et al. (2005). Histopathological examination: Histopathological studies were made in the cecum of chicks were killed at 5 days post infection. Tissues were taken from caecum and fixed in $10 \%$ formalin solution. After fixation, samples were dehydrated in alcohol, cleared in xylene, finally the specimens were embedded in paraffin wax, sectioned at $5 \mu \mathrm{m}$ and stained by haematoxylin and eosin (H\&E), mounted and examined under a light microscope.

\section{Results}

The temporal pattern of oocyst output per day confirms those previously reported (Yunus et al, 2007). Oocyst first appeared on the six days pi, then reached peak on the nine days pi before numbers declined rapidly and the fewest oocysts were detected on 12 days pi. Basically, the same pattern of daily oocyst output was seen in both E. tenella primary and challenge infected chicken, but oocyst output per day and/or as well as totally of the E. tenella challenge infected chicken were significantly lower than E. tenella primary infected chicken (Figure 1). 


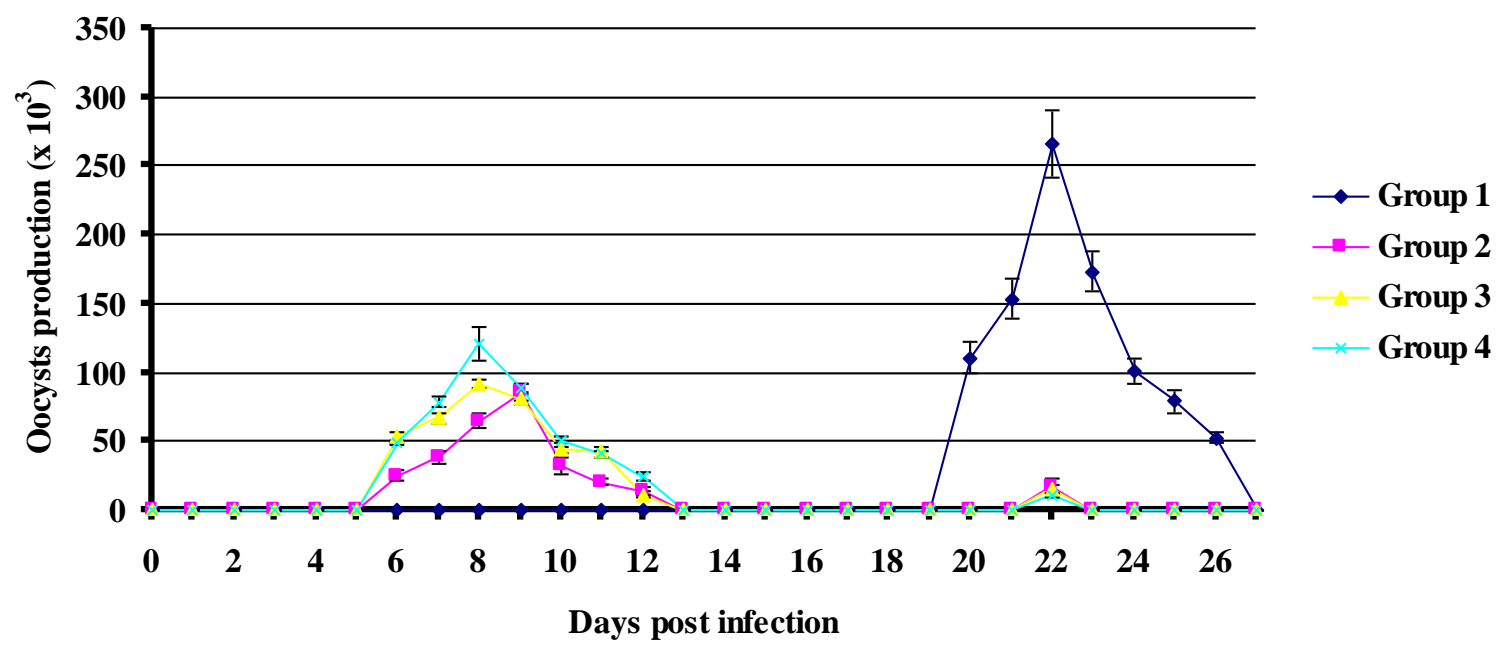

Figure 1: Pattern of daily oocyst production of E. tenella for primary infection and challenge infection of each chicken group, reduction of peak level of oocyst production represented the presence of protective immunity after primary infection, except uninfected chicken group on primary infection (negative control group).

Total of oocyst production in primary infection among chicken groups, group $2[27.8948 \pm 2.13501] \times 10^{4}$, group 3 $[39.1468 \pm 3.06502] \times 10^{4}$ and group $4[45.1928 \pm 2.00045] \times 10^{4}$. On primary infection, there was significantly differences between group 2 and group 3 and 4 ( $<<0.05$ ), while there was not significantly among group 3 and group 4 (Figure 2). Two weeks post primary infection then each chicken groups challenged with $1.0 \times 10^{3} \mathrm{E}$. tenella oocysts and total of oocyst production of each groups were group $1[93.5304 \pm 3.52927] \times 10^{4}$, group $2[8.7548 \pm 1.34584] \times 10^{4}$, group $3[2.8575 \pm$ $0.54535] \times 10^{4}$ and group 4 [1.7085 \pm 0.35456$] \times 10^{4}$ per chick. On challenge test, there was significantly differences total of oocyst production between group 1 and groups $2,3,4(\mathrm{p}<0.01)$, then group 2 was significantly different with group 3 and $4(\mathrm{p}<0.01)$. Furthermore, group 3 and group 4 was not significantly different. Briefly, the pattern of total of oocyst production of each group on primary infection that expressed antigenicity as well as challenge infection that expressed development of protective immunity " immunogenicity " (Figure 2).

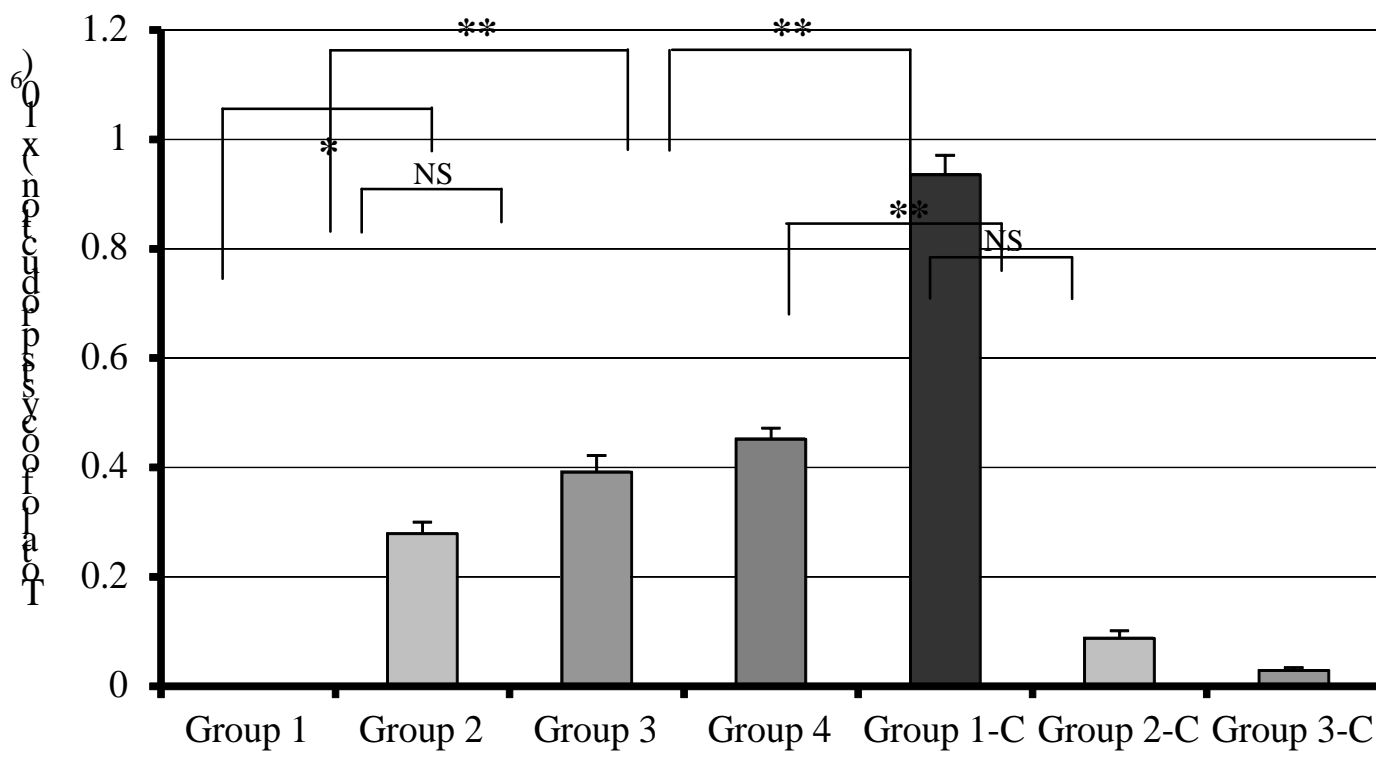

Figure 2: The comparison of total oocysts production of E. tenella between primary infection (initial infection) and challenge infection. Reduction of oocyst production indicated well development of protective immunity after primary infection. Each value represents mean \pm SD of 5 chicks. NS, Not Significant; ** $\mathrm{p}<0.01 ; * \mathrm{p}<0.05$

In this study was occurred reduction of total oocyst production and reproductive index of each group from primary infection to challenge infection (Table 1). Reproductive index of each group on primary infection was group 2 (2789.48), group 3 (1957.34) and group 4 (1506.43), while on challenge infection was group 1 (935.304), group 2 (87.548), group 3 
(28.575) and group 4 (17.085). Clinical signs (such as anemia, anorexia, lethargy) of E. tenella primary infected chicken were very slight, while there was no clinical signs on E. tenella challenge infected chicken, except group 1. Consistency and colour of feces appeared normal in E. tenella challenge infected chicken compared $E$. tenella primary infected chicken. Pathological changes and lesion score of cecum in E. tenella challenge infected chicken were slighter than E. tenella primary infected chicken (Unpublished data).

Table 1: Percentage of reduction of total of oocyst production and reproductive index on challenge infection after primary infection of each chicken group expressed development of protective immunity

\begin{tabular}{|c|c|c|}
\hline \multirow{2}{*}{ Chicken Groups } & \multicolumn{2}{|c|}{ From Primary to Challenge Infection } \\
\hline & $\begin{array}{l}\text { Total Oocyst Production } \\
(\%)\end{array}$ & $\begin{array}{c}\text { Reproductive Index }{ }^{\mathrm{a}} \\
(\%)\end{array}$ \\
\hline Group 1 & \multicolumn{2}{|c|}{$\mathrm{NC}$} \\
\hline Group 2 & \multicolumn{2}{|r|}{96.86} \\
\hline Group 3 & \multicolumn{2}{|r|}{98.54} \\
\hline Group 4 & 96.22 & 98.86 \\
\hline
\end{tabular}

Endogenous development of parasites in E. tenella primary infected chicken occurred well and no inhibition but not so much because low dose E. tenella inoculation (Figure 3). Well development of parasite intracellularly particularly schizonts containing merozoites and immature macrogametocytes in the border epithelial cells of cecum at 120 hours (Figure 3). So, after 72 and 120 hours of E. tenella infection in chicken, moderate inflammatory process can be observed in the lamina propria.

In contrast, endogenous development of E. tenella (schizogony and gametogony) in E. tenella challenged chicken was suppressed and/or uncompleted undergone. Several generations of schizont appeared degenerated consequently schizont not until mature and rupture to release merozoite, damage of cecal mucosa epithelial cell was not occurred and automatically there were no bleeding in cecum (Figure 3). Many abnormal endogenous developments of parasites such as gametogony result in disturbance of syngamy of microgamete and macrogamete. Thus, oocyst formation was not perfectly continued.

The period of five days pi is the time when the second generation schizogony is in progress and most of the third trophozoites and immature schizonts were morphologically degenerated in the chicken challenged E. tenella, whereas in the E. tenella primary infected chicken, the stages of parasite were histologically normal as well as seen multinucleated immature schizonts (Figure 3). In chicken that E. tenella challenged, a few 3rd-generation schizonts remained but morphologically degenerate. In contrast, mature 3rd-generation schizonts were observed within the epithelial cells of primary infected chicken (Figure 3). In the challenge infected chicken, most of the degenerate immature 2nd-generation schizonts had coarse granular cytoplasm and showed poor nuclear differentiation, which made their appearance distinctly different from the normal immature schizonts seen in the primary infected chicken at 5 days pi. Occasionally, at 5 days pi, mature 3rd-generation schizonts had merozoites with a residual body lying free within the parasitophorus vacuole. The mature schizonts in the challenge infected chicken exhibited pycnotic degeneration, while the merozoites in a mature schizont were well developed in the primary infected chicken (Figure 3).
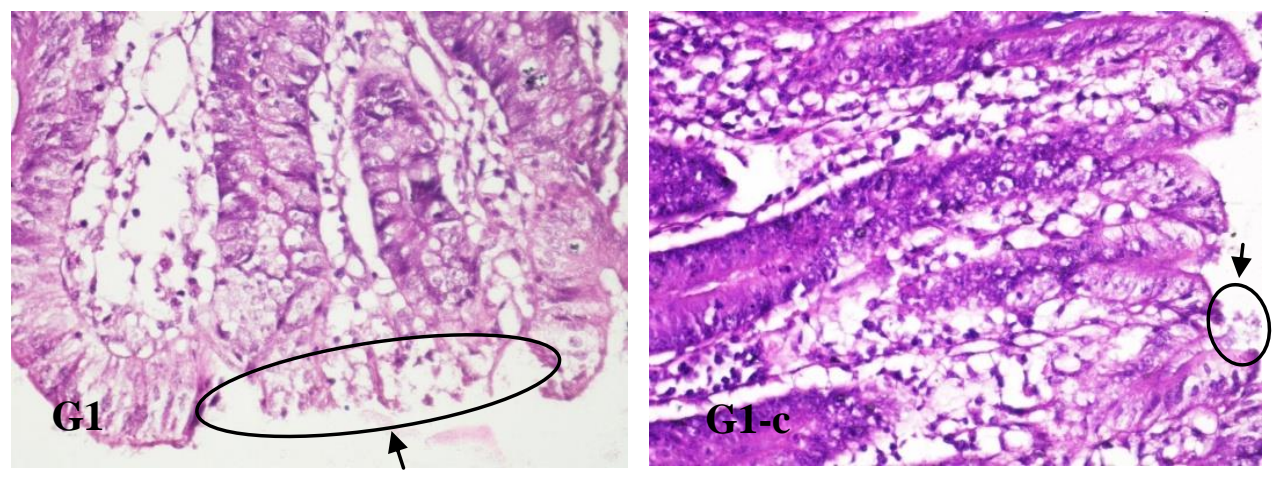

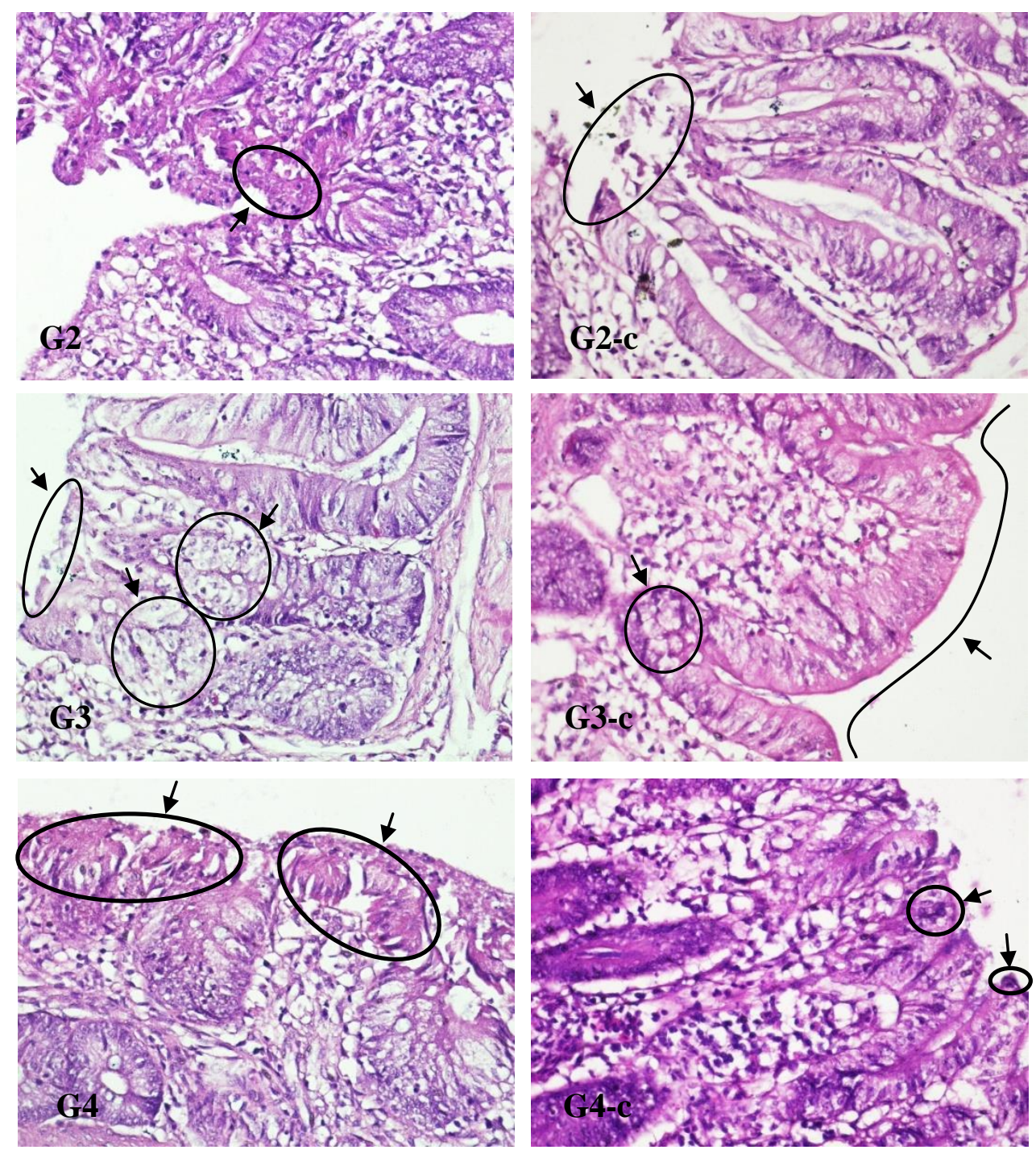

Figure 3: Representative histological images of cecum at 5 days following E. tenella infection (primary and challenge infections). Comparison of morphology of endogenous development stages of E. tenella between primary infection (G1, G2, G3, G4) and challenge infection (G1-c, G2-c, G3-c, G4-c) chicken. G1, part of damaged cecal mucosa and erupted cecal epithelial cells; G1-c, few erupted cecal mucosa post primary infection; G2, bleeding with erythrocytes inside and damaged cecal mucosa; G2-c, limited damaged cecal mucosa epithelial cell and there was degenerated parasite and automatically there were no bleeding in cecum; G3, erupted cecal mucosa epithelial cell; G3-c, most intact cecal mucosa; G4, well development many intracellular schizonts containing merozoites and immature macrogametocytes in the border epithelial cells of cecum; G4-c, schizont appeared degenerated consequently un-break schizont 3 . H\&E stain, objective $\times 40$ (image G1, G1-c, G2, G2-c, G3, G3-c, G4, G4-c). Arrow showed histomorphological endogenous development, bleeding, erupted cecal mucosa and cecal epithelial cells.

\section{Discussion}

The expectation of protective immunity is stimulated after host previously exposured with coccidia then resistant to subsequent infection. The clinical signs exhibited by the chicken during the primary infection such as dehydration, decreased appetite, diarrhea, and also dysentery. In this study, during primary infection, clinical signs were very slight by several low doses. Those signs were not seen during challenge infection, the feces were less well formed for 1 or 2 days after challenge, but diarrhea is never evident.

Infection with one species of Eimeria induces protective immunity in the host that is long lasting and exquisitely specific to that particular parasite (Yun et al, 2000). While a large number of inoculating oocysts is generally required to generate an immune response against Eimeria, some exceptions have been noted, e.g. E. maxima is highly immunogenic and requires only a small number of oocysts to induce almost complete immunity. The early endogenous stages of the parasite life cycle are considered to be more immunogenic than the later sexual stages (Yun et al, 2000) although Wallach et al. 
(1990, 1995) showed that immunization with recombinant gamete associated antigen induced partial protection against challenge infection. Studies using oocysts irradiated to prevent intracellular development, but not invasion, demonstrated partial protection against challenge infection, thereby suggesting that sporozoites may also be immunogenic (Jenkins et al, 1991).

Immunity to Eimeria is stimulated by the initial developing parasite stages, particularly the schizonts, and subsequently boosted and maintained by multiple reexposure to oocysts in the litter. Thus, the recycling of infection following administration of live oocysts is critical for the development of protective immunity (Chapman, Cherry, 1997).

Researchers used different criteria to evaluate coccidial infections. Some suggested that oocyst production might be a very unreliable quantitative criterion (Oikawa et al, 1975) as the number of oocysts produced is affected by factors such as the inherent potential of each species to reproduce in a non-immune host; immunity or resistance developed by the host; the 'crowding' factor; competition with other species of coccidian or other infectious agents; nutrition of the host; and strain differences of the host. The inherent difference in reproductive potential is high for E. tenella and E. acervulina, and low for E. maxima. Immunity, which is specific to each coccidian species, results in decreased production of oocysts after ingestion of infective oocysts (Arabkhazaeli et al, 2011).

The histopathological analyses confirmed more extensive presence of lesions, observed with the light microscope, where more inflammatory cells occur in chickens infected with E. tenella than in other Eimeria species. This criterion was used previously by Karim and Trees (1990) who identified 5 Eimeria species; E. acervulina, E. tenella, E. maxima, E. brunette and E. necatrix; based on a lesion seen at post mortem examinations of naturally infected birds, dimensions of oocyst and lesion seen in experimentally infected chicks with single oocyst. The fact which is agreed with Hein (1971) and Asaduzzaman et al. (2011) E. tenella showed considerable numbers of oocyst in lamina propria of caecum beside severe hemorrhage and complete desquamation of epithelium and edema of muscular tissue which agreed with the finding of Levine (1985).

The entire lamina propria revealed severe haemorrhages, necrosis and disintegration of glandular epithelial cells. Several schizonts were observed in the epithelial cells along with merozoites, infiltrating neutrophils and eosinophils.

Ruff (1999) determined that each oocyst ingested by a host has the potential to give rise to hundreds of thousands of oocysts within the feces after seven to twelve days. It was discovered that daily inoculations of small numbers of oocysts over twenty days produced a stronger immunity than when a large number of oocysts was given in a single dose (Reid, 1990). This discovery was labeled as a trickle infection. The trickle infection suggests that for chickens Eimeria species protective immunity is developed only after the bird has been infected by the parasite several times through cycling. Beattie (1998) it was generally found that the motile sporozoites played an important role in conferring an immune response while the gametogonic stages elicited little protective immunity. Protective immunity is generally regarded as the prevention of oocyst production and absence of clinical signs in birds challenged by the parasites (Beattie, 1998). Once protective immunity is developed for a certain Eimeria species (and sometimes strain) the bird is immune against further infection with this parasite (Lightowlers, 1994). Eimeria maxima best demonstrates acquired immunity; a single infection initiated with only a few oocysts results in the development of almost complete (>99.99\%) immunity (Smith et al, 2002).

\section{Conclusion}

The low number virulent $E$. tenella had low reproductive index and few histopathological changes effect that represents a promising strategy to prevent cecal coccidiosis in chickens.

Conflict of Interest: The authors declare that there is no competing interest among them.

\section{Acknowledgment}

This study was supported by Directorate of Higher Education, Ministry of Education and Culture of the Republic of Indonesia for funding through higher education excellence research grant for a research contract number 304/SP2H/LT/DRPM/III/2016.

\section{References}

1. Arabkhazaeli F, Nabian S, Modirsaneii M, Mansoori V, Rahbari S. (2011). Biopathologic characterization of three mixed poultry Eimeria spp. Isolates. Iran J Parasitol; 6: 23-32.

2. Asaduzzaman M, Miah MS, Siddika A, Popy N, Hossain MM. (2011). Experimental production of necrotic enteritis in broiler chickens. Bangladesh J Vet Med; 9: 33-41.

3. Beattie SE. (1998). Immunity to and transport of sporozoites of Eimeria species in the domestic fowl (Gallus domesticus). University of Guelph MSc thesis, 3-12.

4. Chapman HD, Cherry TE. (1997). Eye spray vaccination: infectivity and development of immunity to Eimeria acervulina and Eimeria tenella. J Appl Poultry Res; 6: 274-278. 
5. Dalloul RA, Lillehoj HS. (2006). Poultry coccidiosis: recent advancements in control measures and vaccine development. Exp Rev Vaccines; 5: 143-163.

6. Haug A, Thebo P, Mattsson JG. (2007). A simplified protocol for molecular identification of Eimeria species in field samples. Vet Parasitol; 146: 35-45.

7. Hein H. (1971). Pathogenic effects of Eimeria necatrix in young chickens. Exp Parasitol; 30: 321-330.

8. Jenkins MC, Augustine PC, Danforth HD, Barta JR. (1991). X-irradiation of Eimeria tenella oocysts provides direct evidence that sporozoite invasion and early schizont development induce a protective immune response(s). Infec Immun; 59: 4042- 4048.

9. Karim MJ, Trees AJ. (1990). Isolation of five species of Eimeria from chicken in Bangladesh. Trop Anim Health Prod; 22: 153-159.

10. Lightowlers MW. (1994). Vaccination against animal parasites. Vet Parasitol; 54: 177-204.

11. Levine ND. (1985). Erhardorina n.g, Ascogregarina polynesiensis n. spp., Eimeria golemanskiin.spp., Isospora tamariscinin. spp., Gregarina kazumii n. nom., new combinations and emendations in the names of apicomplexan protozoa. J Protozool; 32: 359-63.

12. Oikawa H, Kawaguchi H, Nakamoto K, Tsunoda K. (1975). Field surveys on coccidial infection in broilers in Japanresults obtained in autumn and winter and summarized in 1973. Nihon Juigaku Zasshi; 37: 271-279.

13. Reid WM. (1990). History of avian medicine in the United States. x. control of coccidiosis. Avian Dis; 34: $509-525$.

14. Ruff MD. (1999). Important parasites in poultry production systems. Vet Parasitol; 84: 337-347.

15. Smith AL, Hesketh P, Archer A, Shirlery MW. (2002). Antigenic diversity in Eimeria maxima and the influence of host genetics and immunization schedule on cross-protective immunity. Infect Immun; 70: 2472-2479.

16. Soulsby E. (1986). Helminths, Arthropods and Protozoans of Domesticated Animals. $7^{\text {th }}$ Ed., Bailliere Tindall, London, UK.

17. Wallach M, Pillemer G, Yarus S, Halabi A, Pugatsch T, Mencher D. (1990). Passive immunization of chickens against Eimeria maxima infection with a monoclonal antibody developed against a gametocyte antigen. Infect Immun; 58: 557- 562.

18. Wallach M, Smith NC, Petracca M, Miller CM, Eckert J, Braun R. (1995). Eimeria maxima gametocyte antigens: potential use in a subunit maternal vaccine against coccidiosis in chickens. Vaccine; 13: 347-354.

19. Yun CH, Lillehoj HS, Lillehoj, EP. (2000). Intestinal immune responses to coccidiosis. Develop Comp Immunol; 24: 303-324.

20. Yunus M, Horii Y, Makimura S, Smith AL. (2005). The Relationship Between the Anticoccidial Effects of Clindamycin and the Development of Immunity in the Eimeria pragensis/Mouse Model of Large Intestinal Coccidiosis. J Vet Med Sci; 67: 165-70.

21. Yunus M, Suwanti LT, Mufasirin (2007). Cecal Goblet Cell Response on Intracellular Development of Eimeria tenella in Susceptible and Infected Chickens. Vet Med J; 23: 132-138. 